\title{
A randomized control trial of single dose versus multiple doses of IV antibiotic prophylaxis in caesarean delivery
}

\author{
M Vathana ${ }^{\mathrm{a}}$, K Muhunthan ${ }^{\mathrm{b}}$
}

\begin{abstract}
Objectives

To determine the effectiveness and safety of single doses antibiotic against conventional multiple doses regimen on clinically detectable maternal and neonatal infectious morbidity.

\section{Method}

This is a randomized, non-blind clinical trial on women undergoing caesarean section. By block random sampling, 369 women, who were enrolled in this study, 185 (50.1\%) randomly received single dose of antibiotics and 184 (49.8\%) received multiple postoperative doses of antibiotics. All potentially infected cases were excluded. These patients were followed up prospectively for infectious and neonatal complications till discharge and verbal enquiry or direct observation done during suture removal. The effectiveness was measured in terms of febrile morbidity, surgical site infection, endometritis, urinary tract infection, other infection along with duration of hospital stay. Chi-square analysis (Fisher's Exact
\end{abstract}

Test) of variance were performed with equivalence margin was set at $5 \%$ ( $p$ value).

\section{Results}

The incidence rates of post-caesarean infections were $1.8 \%$ and $3.2 \%$ in single dose and multiple dose regimens respectively with the incidence rate ratio of 0.3 [95\% Cl 0.065-1.63) p-value=0.284]. There were no statistically significant differences in febrile morbidity $(p=0.28)$, wound infections $(p=0.123)$, perinatal outcome $(p>0.05)$ and median duration of hospital stay $(p=0.329)$ in both arms.

\section{Conclusions}

Single combined prophylactic antibiotic usage immediately after cord clamping is equally effective as multiple conventional regimen following the caesarean deliveries in prevention of infectious morbidities and duration of hospital stay, with benefit of reducing staff workload along with reduced medication costs and the emergence of drug selective resistant bacteria.

Key words: Caesarean section, surgical wound infection, antibiotic prophylaxis

Sri Lanka Journal of Obstetrics and Gynaecology

2018; 40: 92-100

DOI: http://doi.org/10.4038/sljog.v40i4.7871

a Acting Consultant Obstetrician and Gynaecologist, District General Hospital, Mannar, Sri Lanka

b Senior Lecturer, Department of Obstetrics \& Gynaecology, Faculty of Medicine, University of Jaffna, Sri Lanka

Correspondence: MV, e-mail: vathanama@gmail.com>

Received 19 September 2018 and revised version accepted 1 November 2018.

http://orcid.org/0000-0002-7388-3794

Competing interest: The authors report no conflict of interest

This is an open-access article distributed under the terms of the Creative Commons Attribution 4.0 International License, which permits unrestricted use, distribution and reproduction in any medium provided the original author and source are credited. 


\section{Introduction}

Infection is one of the most common complications of caesarean delivery. Women undergo caesarean section have a 5-20 fold greater chance of getting an infection compared with women who give birth vaginally ${ }^{1}$. Usage of prophylactic antibiotic is proved to be effective in lowering post-operative infections ${ }^{2-4}$. The $60-70 \%$ reduction in endometritis and the $30-65 \%$ reduction in wound infection rate prompted the Cochrane library to recommend prophylactic antibiotics to women who undergo both elective and non-elective caesarean delivery ${ }^{1}$. The antibiotic prophylaxis in women who undergo caesarean delivery also has been proven to be beneficial in decreasing infectious morbidities and length of hospital stay5.

The potential benefit from prophylactic antibiotic should always be balanced against the possible damage like drug resistance. Now it has been much concerned globally and nationally regarding the misuse of antibiotics leading to a high incidence of resistance, cost effectiveness or suboptimal treatments ${ }^{6-7}$.

An attempt to reduce antibiotic usage is timely necessary because injudicious use of antibiotics not only adds to financial burden, burden on hospital resources in terms of human resource, time and equipment, but also exposes women to undesirable side effects ranging from mild nausea and rash to severe life threatening drug reactions. More over the antibiotics compromise immunity suppress host defenses and subject to further risk of acquiring infections. The duration of prophylaxis should be as short as it could be, in order to avoid the danger of occurrence of resistant strains and to avoid unnecessary patient discomfort and unnecessary expenses. Hence serious efforts should be made to minimize unnecessary prolongation of antibiotics.

Given the ever-increasing economic and health burden of caesarean deliveries in Sri Lanka and the need for convenient and less costly regimens, this study was designed to compare the effectiveness of single dose of cefuroxime plus metronidazole and conventional multi-dose regimen for infection prophylaxis in caesarean section.

\section{Methodology}

This was a randomized controlled, non-blind trial, which was carried out in the Teaching Hospital Jaffna. Study population was women undergoing caesarean delivery - both elective and non-elective and had consented for the study were eligible for inclusion, but the study exclude those with history of allergy, prior antibiotic usage within two weeks, pyrexia $>38^{\circ}$ Celsius or obvious evidence of clinical infection or chorioamniotis, known medical illness that might cause pyrexia or separate indication for use of antibiotics [known immunodeficiency syndromes, chronic disease of cardiovascular, renal, hepatic or gastrointestinal system or severe anemia $(\mathrm{Hb}<8 \mathrm{~g} / \mathrm{dl})]$.

The primary outcome measured was clinically detectable postoperative infectious morbidity, defined as fever, wound infection, endometritis, urinary tract infection or serious infection (such as bacteremia, septic shock, septic thrombophlebitis, necrotizing fasciitis and death) after caesarean section.

The duration of hospital stay, adverse events of treatment (eg allergic reactions, antibiotic-associated diarrhea, development of bacterial resistance and any infant outcomes reported are considered as secondary outcome.

Procedure: Stratified block randomization was done by using computer generated table and sequentially numbered sealed opaque envelopes coded with the appropriate treatment regimen groups (group A - only single dose of IV cefuroxime 1.5g and IV Metronidazole $500 \mathrm{mg}$ immediately after cord clamping and group B (multiple dose) - IV cefuroxime 750 tds and Metronidazole $500 \mathrm{mg}$ tds were continued for up to 24 hours following the caesarean section) was prepared by the supervisor (not by principal investigator). Each mother was randomized into two by the investigator according to the predetermined randomized allocation sequence.

Post operatively, group B mothers were allowed oral cefuroxime $750 \mathrm{mg}$ tds and Metronidazole 400mg tds for 7 days completion, within 6 hours, mobilized out of bed within 12 hours. Urinary catheter was removed within 12-24 hours. Dressings were removed in 36-48 hours followed by bath. Full blood count was checked on second post-operative day. Education regarding nutrition, hygiene and wound care were given to all. Uncomplicated mothers were discharged on third post-operative day with verbal and written advice to report if sign or symptom of infection developed. They were called for follow up and stitch removal (if prolene) 
on seventh to eighth post-operative day. Later until postpartum completions of 42 days all mothers were followed up weekly through over the phone.

If any mother who developed post-operative febrile morbidity, wound infection, endometritis, urinary tract infection, pneumonia or other serious infection were documented and treated promptly.

Sample size: We liked to estimate the population proportion with a tolerance of $(\mathrm{e})=0.02$.

If we assumed surgical side infection rate after caesarean section is 0.1 as it is prior information 20 with $95 \%$ confidence interval

$\mathrm{N}=\mathrm{Z} / 2$ * $\mathrm{P}(1-\mathrm{P})$ where $\mathrm{N}=\mathrm{n} 1+\mathrm{n} 2$ e 2

$=1.96 *(0.1) *(0.9)(0.02) 221$

$=441$

( $\mathrm{n} 1=221$ and $\mathrm{n} 2=221)$

Total number of women required for the study was 442.

This was inflated by 20 to accommodate any dropouts or crossover between groups.

Therefore total number of women required for the study was 530 .

Statistical analysis: Data were statistically described in terms of mean +/- standard deviation (+/- SD), median and interquartile range, or frequencies (number of cases) and percentages when appropriate. Comparison of numerical variables between the study groups was done using $t$ test for independent samples. For comparing categorical data, Chi square $\left(\chi^{2}\right)$ test was performed. Fisher's exact test was used instead when the frequency was less than 5 . p value less than 0.05 was considered statistically significant. All statistical calculations were done using computer programs SPSS (Statistical Package for the Social Science; SPSS Inc., Chicago, IL, USA) version 22 for Microsoft Windows.

The study was approved by the Ethical Review Committee of University of Jaffna and an informed consent was obtained from all women who agreed to participate in the study.

\section{Results}

A total of 465 pregnant women were assessed for eligibility. Of these, 90 participants were excluded from the study according to the exclusion criteria. Out of eligible 375 pregnant women 188 randomly received single dose regime and 187 received multiple dose regime antibiotic for 7 days (dose regime has been explained in methodology). 2 participants in single dose regime declined to participate post operatively and 4 participants from both arm lost the follow-up (Figure 1).

There is homogeneity among the study participants in terms of their baseline characteristics in both arms (p-value >0.05) Table 1.

As showed in Table 2 there were no statistically significant differences in outcome parameters between the two treatment groups. Primary outcome postoperative infections developed in $8(2.1 \%)$ patients $\mathrm{p}=0.284$, among them two patients $(1.8 \%)$ were from the single dose group and six patients (3.2\%) from the multiple dose group.

Three patients (1.6\%) in the multiple dose group developed wound infection, but none of the patients in single dose group developed wound infection during the study period. All are culture positive superficial infections and treated according to sensitivity tests. None of these differences were statistically significant.

There were no reported culture positive endometritis, urinary tract infection or other evidence of infection. None of the patients suffered adverse drug effects during our study period. Perinatal out come as recorded in the hospital discharge record, showed no statistically significant differences between the two treatment groups.

Variables that were associated with any infectious morbidity are shown in Table 3. It disclosed statistical significance of important risk factors for post-operative infection.

Performance under general anesthesia (OR 10.2, 95\% 10.2-99.08, $\mathrm{p}=0.124$ ), corticosteroid exposure (OR 2.19, 95\% CI0.09-2.33, $\mathrm{p}=0.295$ ), usage of nylon for skin closure (OR 3.7, 95\% CI: 0.90-15.1, $\mathrm{p}=0.073$ ), interrupted suturing technique (OR 1.4, 95\% CI: 0.247.57, $\mathrm{p}=0.643$ ) appear to be risk factor; 


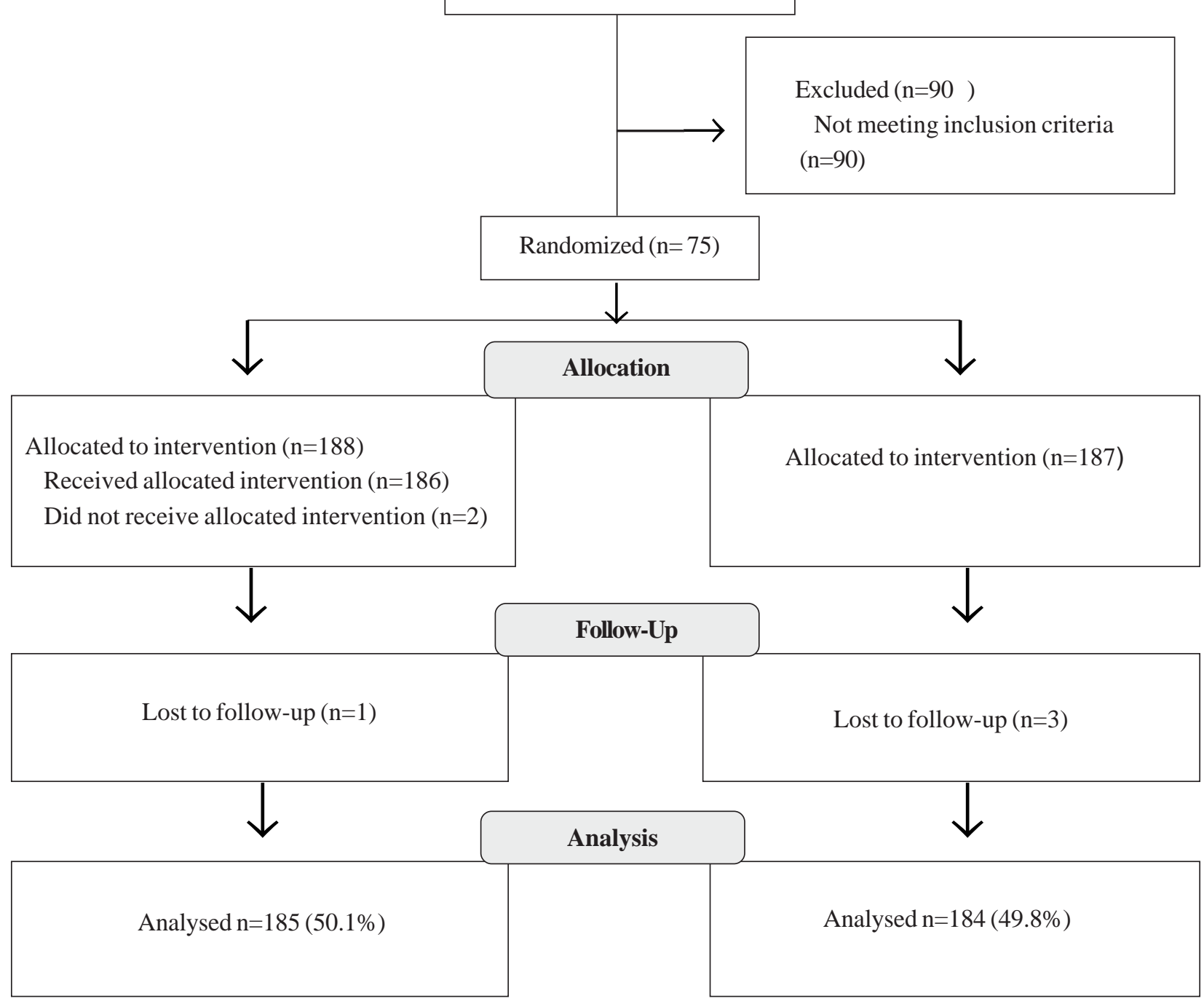

Figure 1. Flow diagram of the study participant.

Table 1. Distribution of characteristics in study participants

\begin{tabular}{|l|l|c|c|}
\hline \multirow{2}{*}{ Variable } & \multicolumn{2}{|c|}{ Randomized study group } & \multirow{2}{*}{ P - value } \\
\cline { 2 - 3 } & $\begin{array}{c}\text { Single dose arm } \\
\mathrm{n}=185(50.1 \%)\end{array}$ & $\begin{array}{c}24 \text { hours usage arm } \\
\mathrm{n}=184(49.8 \%)\end{array}$ & \\
\hline Age (years) mean +/- SD & $29.72 \pm 5.012$ & $29.46 \pm 5.389$ & 0.639 \\
BMI (Kg/m²), mean+/- SD & $26.44 \pm 4.169$ & $25.96 \pm 3.933$ & 0.261 \\
Gestational age (wks), mean +/- SD & $38.9+-1.55$ & $38.9+-1.75$ & 0.802 \\
Multiparity, n (\%) & $81(44.02 \%)$ & $69(37.5 \%)$ & 0.244 \\
VE>/= 6 n (\%) & $34(18.3 \%)$ & $36(19.5 \%)$ & 1.000 \\
& & & Continued \\
\hline
\end{tabular}




\begin{tabular}{|c|c|c|c|}
\hline \multirow[t]{2}{*}{ Variable } & \multicolumn{2}{|c|}{ Randomized study group } & \multirow[t]{2}{*}{$P$ - value } \\
\hline & $\begin{array}{l}\text { Single dose arm } \\
n=185(50.1 \%)\end{array}$ & $\begin{array}{c}24 \text { hours usage arm } \\
n=184(49.8 \%)\end{array}$ & \\
\hline ROM $>/=6 h, \mathrm{n}(\%)$ & $30(16.2 \%)$ & $29(15.7 \%)$ & 0.948 \\
\hline Type of CS- Non elective n (\%) & $69(37.2 \%)$ & $71(38.5 \%)$ & 0.371 \\
\hline Anesthesia- GA, n (\%) & $3(1.6 \%)$ & $3(1.6 \%)$ & 0.655 \\
\hline Duration of surgery > $30 \mathrm{~min}, \mathrm{n}(\%)$ & $51(27.5 \%)$ & $47(25.5 \%)$ & 0.724 \\
\hline Grade of the operator & & & \\
\hline Consultant & $9(4.8 \%)$ & $7(3.8 \%)$ & 0.799 \\
\hline Blood loss >500ml, n (\%) & $6(3 \%)$ & $1(0.5 \%)$ & 0.12 \\
\hline BW of the baby $(\mathbf{k g})$, mean $+/-$ SD & $2.84+-0.66$ & $2.9+-0.61$ & 0.827 \\
\hline Type of pregnancy (in \%) & & & \\
\hline Singleton & 178 & 178 & 1.000 \\
\hline
\end{tabular}

$\mathrm{n}$ - number, SD - standard deviation, VE - vaginal examination, ROM - rupture of membrane, CS - cesarean section, post op - post operative, IQR - interquatile range, BW - birth weight of the baby.

Table 2. Distribution of post-operative infectious morbidity in study participants

\begin{tabular}{|l|l|l|l|}
\hline \multirow{2}{*}{ Post-operative outcome } & \multicolumn{2}{|c|}{ Randomized study group } & \multirow{2}{*}{ - value } \\
\cline { 2 - 3 } & $\begin{array}{c}\text { Single dose arm } \\
\mathrm{n}=185(50.1 \%)\end{array}$ & $\begin{array}{c}24 \text { hours usage arm } \\
\mathrm{n}=184(49.8 \%)\end{array}$ & \\
\hline All post op. infection, n (\%) & $2(1.8 \%)$ & $6(3.2 \%)$ & 0.284 \\
Wound infection, n (\%) & $0(0 \%)$ & $3(1.6 \%)$ & 0.123 \\
Endometritis, n (\%) & $0(0 \%)$ & $0(0 \%)$ & \\
UTI, n (\%) & $0(0 \%)$ & $0(0 \%)$ & \\
Other infection, n (\%) & $0(0 \%)$ & $0(0 \%)$ & \\
Adverse drug reaction, n (\%) & $0(0 \%)$ & $11(5.9 \%)$ & 0.629 \\
Neonatal Outcome & $10(5.4 \%)$ & $6(3.2 \%)$ & 0.791 \\
NICU admission & $4(2.1 \%)$ & $2(1 \%)$ & 0.369 \\
Neonatal death & $1(0.5 \%)$ & $3(2-2)$ & \\
Still birth / IUD & $4(2-2)$ & & \\
Duration of post op. hospital stay, & & & \\
median (IQR) & & & \\
\hline
\end{tabular}

$\mathrm{n}$ - number, UTI - urinary tract infection, NICU - neonatal intensive care unit, IUD - death in utero, IQR - interquartile range. 
Table 3. Distribution of risk factors among arms complicated with infectious morbidity and non-complicated arm $[(n=8) p=0.284$, IRR $=0.395 \% \mathrm{CI}=0.06-1.63]$

\begin{tabular}{|c|c|c|c|c|c|}
\hline \multirow[t]{2}{*}{ Variables } & \multicolumn{2}{|c|}{ Infectious morbidity } & \multirow[t]{2}{*}{ P value } & \multirow[t]{2}{*}{ OR } & \multirow[t]{2}{*}{$95 \%$ CI } \\
\hline & Not complicated & Complicated & & & \\
\hline \multicolumn{6}{|c|}{ Corticosteroid usage } \\
\hline Not given & 314 & 6 & 0.295 & 2.19 & $0.09-2.33$ \\
\hline Given & 48 & 2 & & & \\
\hline \multicolumn{6}{|c|}{ Vaginal Examination } \\
\hline$<6$ or not done & 69 & 1 & 0.526 & 0.59 & $0.2-13.76$ \\
\hline$>/=6$ & 292 & 7 & & & \\
\hline \multicolumn{6}{|l|}{ ROM } \\
\hline$<6 \mathrm{~h}$ or intact & 303 & 7 & 0.627 & 0.749 & $0.09-6.2$ \\
\hline$>/=6 \mathrm{~h}$ & 58 & 1 & & & \\
\hline \multicolumn{6}{|l|}{ Type of CS } \\
\hline Elective CS & 219 & 6 & 0.715 & 0.5 & $0.109-2.75$ \\
\hline Non Elective CS & 142 & 2 & & & \\
\hline \multicolumn{6}{|l|}{ MOA } \\
\hline RA & 356 & 7 & 0.124 & 10.2 & $10.2-99.08$ \\
\hline GA & 5 & 1 & & & \\
\hline \multicolumn{6}{|l|}{ Operator grade } \\
\hline Consultant & 16 & 1 & 0.316 & 0.3 & $0.03-2.76$ \\
\hline MO & 346 & 7 & & & \\
\hline \multicolumn{6}{|l|}{ Blood loss } \\
\hline$<500 \mathrm{ml}$ & 354 & 8 & 1.000 & 0.97 & 0.96-0.99 \\
\hline$>500 \mathrm{ml}$ & 7 & - & & & \\
\hline \multicolumn{6}{|c|}{ Duration of surgery } \\
\hline$<30 \min$ & 263 & 8 & 0.116 & 0.971 & 0.95-0.99 \\
\hline$>30 \min$ & 98 & 0 & & & \\
\hline \multicolumn{6}{|l|}{ Skin closure } \\
\hline Sub cuticle & 296 & 6 & 0.643 & 1.4 & $0.24-7.57$ \\
\hline Interrupted & 66 & 2 & & & \\
\hline Suture material & & & & & \\
\hline Vicryl & 285 & 4 & 0.073 & 3.7 & $0.90-15.1$ \\
\hline Nylon & 77 & 4 & & & \\
\hline
\end{tabular}

incidence rate ratio (IRR), CS - cesarean section, RA - regional anesthesia, GA - general anesthesia, $\mathrm{h}$ - hours, min - minute, MO - medical officer, ROM - rupture of membrane, CI - confident interval, OR - odd ratio. MOA - mode of anesthesia. 


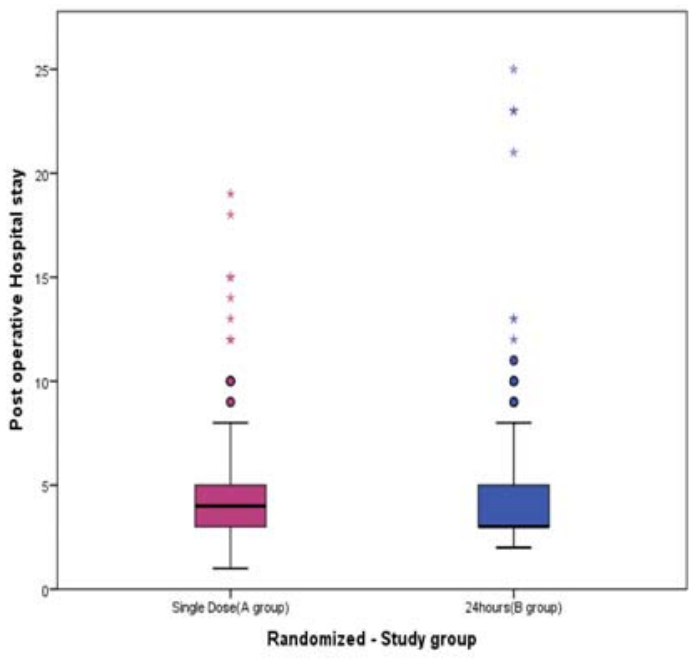

Figure 2. Distribution of post-operative hospital stay.

Except performance under general anesthesia, others were not significant statistically.

But ruptured membrane more than six hours (OR 0.749, 95\% CI: 0.09-6.2, p=0.627), six or more vaginal examinations prior to the cesarean (OR 0.59, 95\% CI: 0.2-13.76, $\mathrm{p}=0.526$ ), non-elective cesarean (OR 0.5, 95\% CI: 0.109-2.75, $\mathrm{p}=0.715$ ), and blood loss greater than $500 \mathrm{ml}$ (OR 0-97, 95\% CI: 0.96-0.99, p=1), operator grade (OR 0.3,95\% CI0.03-2.76, $\mathrm{p}=0.316$ ) appear to be protective without any statistical significance.
The duration of hospitalization was the same in the two groups of patients, with a median stay of four days for the single dose group and three days for the multiple dose group without any statistically significant difference $\mathrm{p}=0.329$.

\section{Discussion}

Prophylactic antibiotics are given to help prevent infection. Burke and colleagues in 1961 were the first to describe the role of antibiotics in reduction of rates of wound infection in animal models when administrated before skin contamination ${ }^{8}$. The later development regarding benefits of using prophylactic antibiotics in the prevention of serious infection and febrile morbidity after cesarean section have been well documented in the literature. But the recommendations about single-dose prophylaxis are not uniformly practiced.

In the multicenter trials evaluated by Hopkins L, Smaill $\mathrm{F}$ in the Cochrane review compared various trials that compared different antimicrobial agents, comparison between the routes and the number of doses of drugs given $^{9}$. The Table 4 shows comparison of any single dose systemic regimen (pre, post, intra-operative) vs. any multiple dose regimen in terms of wound infections.

Table 4. Comparison of wound infections in other studies

\begin{tabular}{|lccc|}
\hline Study & Treatment n/N (\%) & Control n/N (\%) & Peto odds ratio \\
\hline Galask & $4 / 162(6.4 \%)$ & $4 / 79(5 \%)$ & 0.45 \\
Roex & $7 / 66(4.2 \%)$ & $2 / 72(2.7 \%)$ & 3.58 \\
Tassi & $3 / 100(3 \%)$ & $1 / 100(1 \%)$ & 2.67 \\
Varner & $3 / 20(15 \%)$ & $1 / 9(11 \%)$ & 1.37 \\
Von Mandach & $17 / 536(3.1 \%)$ & $20 / 516(3.8 \%)$ & 0.81 \\
Jakobi & $0 / 50(0 \%)$ & $1 / 50(2 \%)$ & 0.14 \\
Hawrylyshyn & $1 / 64(1.5 \%)$ & $1 / 60(1.6 \%)$ & 0.94 \\
Hartert & $1 / 81(1.2 \%)$ & $0 / 58(0 \%)$ & 5.56 \\
McGregor & $4 / 46(8.6 \%)$ & $4 / 24(16 \%)$ & 0.46 \\
Mc Gregor & $5 / 195(2.5 \%)$ & $3 / 91(3.2 \%)$ & 0.76 \\
Parsons & $0 / 90(0 \%)$ & $1 / 62(1.6 \%)$ & 0.09 \\
Present & $16 / 300(5.6 \%)$ & $14 / 300(4.6 \%)$ & 0.857 \\
\hline
\end{tabular}


Although antibiotic used and route of administration varies in different above studies, the overall results indicated that multiple dose does not offer any added benefit when compared with single dose regimen which is similar to our study primary outcome evaluated in Table 2.

Hospital stay was almost the same in both groups which means that single dose versus multiple doses of antibiotic does not affect the hospital stay and is related to number of days required for wound healing. This has also been confirmed by Tchabo et al who reported no significant difference in the incidence of postoperative infection and mean duration of hospital stay when compared single dose antibiotic verses multiple dose antibiotics ${ }^{10}$.

Regarding neonatal outcomes, we found there was no statistical significant difference between both groups in the occurrence of clinically detectable neonatal death, still birth or neonatal ICU admission. Similar results was reported by Thigpen et al. 2005 ${ }^{11}$, Hamit et al. $2009^{12}$, and Sullivan et al. $2009^{13}$.

Our study findings are corroborated by previous studies, which show that single dose antibiotic regimens are as effective as conventional multiple doses for prevention of infectious morbidity following cesarean section. This study did not address the costanalysis of cefuroxime and metronidazole and their potential for emergence of resistant organisms. Both are vast areas, not within the scope of this small clinical trial.

\section{Conclusion and recommendations}

Since the differences in the rates of febrile morbidity and wound infection were not statistically significant, and there were no urinary tract infection, endometritis or other infections in women who received either single dose or multiple doses of prophylactic antibiotics for caesarean section in this study, it may be argued that both single and multiple dose regimen protected equally against post-caesarean section infectious morbidity and that a single prophylactic dose prevents unneces-sary long course of antibiotics and susceptibility to antibiotic resistance.

Though the objective of this study did not include cost effectiveness of either regime, the cost of antibiotic therapy is apparently reduced in the single dose group. Therefore, when considering medication cost and drugsusceptibility pattern of the common bacteria causing surgical-site infection in our setting, single dose regime for prophylaxis is the rational choice. However, prophylactic antibiotics should not replace proper preand intra operative preparation and meticulous surgical technique.

In addition, the use of single dose regime reduces workload to the nurses, especially at night when few nurses are on duty in the ward. The shortage of health care workers is a well-known problem in many resource limited settings especially in peripheral units of our country.

\section{Limitations}

All participates called for follow up and stitch removal (if prolene) on seventh to eighth post-operative day. Later until postpartum completions of 42 days all mothers were followed up weekly through over the phone.

We were unable to follow up the patient directly until the whole postpartum period.

\section{Acknowledgements}

I would like to thank all of the medical staff of Teaching Hospital Jaffna and all women who agreed to participate this study.

\section{References}

1. Smaill F, Gyte G. Antibiotic prophylaxis versus no prophylaxis for preventing infection after cesarean section. Cochrane Database of Systematic Reviews 2010, Issue 1. Art. No.: CD007482. DOI: 10.1002/ 14651858.CD007482.pub2

2. Antibiotic prophylaxis for surgery. Treatment guidelines. The Medical Letter 2004; 2(20): 27-32.

3. Antimicrobial prophylaxis in surgery. The Medical Letter 2001; 43: 92-8.

4. ASHP therapeutic guidelines on antimicrobial prophylaxis in surgery. AJHP 1999; 56: 1839-87.

5. Killian C, Graffunder E, Vinciguerra T, Venezia R. Risk factors for surgical site infections following cesarean section. Infect Control Hosp Epide moil 2001; 22(10): 613-7. 
6. Carey B, Cryan B. Antibiotic misuse in the community - a contributor to resistance? Ir Med J 2003 Feb; 96 (2): 43-44, 46.

7. Klem C, Dasta JF. Efforts of pharmacy to reduce antibiotic resistance. New Horiz 1996 Aug; 4(3): 377-84.

8. Burke JF. The effective period of preventive antibiotic action in experimental incisions and dermal lesions. Surgery 1961; 50: 161-8.

9. Hopkins L, Smaill F. Antibiotic prophylaxis regimens and drug for caesarean section. Cochrane Database Syst Rev 2000; 2: CD001136.

10. Tchabo JG, Cutting ME, Butter C. Prophylactic antibiotic in patients undergoing total vaginal or abdominal hysterectomy. Int Surg 1985; 70 : 349-52.
11. Thigpen B, Hood WA, Chauhan S, Bufkin L, BoWll S, Magann E, Marrison SC.Timing of prophylactic antibiotic administrationin the uninfected laboring gravida: a randomized clinical trial. Am J Obstet Gynecol 2005; 192(6): 1864-8; discussion 186871.

12. Hamit $Z$ et al. When should we perform prophylactic antibiotics in elective Cesarean cases? Arch Gynecol Obstet 2009; 280: 13-18.

13. Sullivan SA, Simith T, Chang E, Hulsey T, Vandorsten JP, Soper D. Administration of cefazolin prior to skin incision is superior to cefazolin at cord clamping in preventing post Cesarean infectious morbidity: a randomised, controlled trial. Am J Obstet Gynecol 2007; 196(5): 455.e1-5. 